\title{
Spiritual Intelligence and Post-abortion Depression: A Coping Strategy
}

Farnoosh Moafi ${ }^{\mathrm{a}}$, Maryam Momeni ${ }^{\mathrm{b}}$, Mohadeseh Tayeba ${ }^{\mathrm{a}}$, Sarah Rahimi ${ }^{\mathrm{b}}$ \& Hamideh Hajnasiri ${ }^{\mathrm{a}}$

${ }^{a}$ Midwifery Department, Qazvin University of Medical Sciences, Qazvin, Iran

${ }^{\mathrm{b}}$ Nursing Department, Qazvin University of Medical Sciences, Qazvin, Iran

\begin{abstract}
Loss of pregnancy is a major stressor which can cause mental disorders. In stressful conditions, spiritual intelligence can be used as a coping strategy to manage and cope with the stressor. It may also produce positive outcomes in spontaneous abortion. This study aimed to assess the relationship of spiritual intelligence with depression after spontaneous abortion. This crosssectional descriptive-correlational study was done in 2015-2016 on 185 women with spontaneous abortion who were hospitalized in the postnatal care ward of Kowsar teaching hospital, Qazvin, Iran. Data collection was performed in two steps. In the first step, a demographic and obstetric characteristics questionnaire and the Spiritual Intelligence Self-Report Inventory were completed by participants during their hospital stay. In the second step, i.e., 7 days after spontaneous abortion, the Edinburgh Postnatal Depression Scale was completed for each participant over the phone. Variables which were found in univariate analysis to have a significant correlation with depression were entered into multiple logistic regression analysis to assess their roles in predicting depression. Most women were depressed (54\%). Multiple logistic regression analysis revealed that the significant predictors of depression were women's educational status (OR 0.419; CI 0.18-0.93), gestational age at the time of abortion (OR 1.121; CI 1.02-1.22), and the personal meaning production dimension of spiritual intelligence (OR 0.82; CI 0.73-0.91). Spiritual intelligence is significantly correlated with post-abortion depression. Developing and following strategies for promoting spiritual intelligence can alleviate depression, stress, and anxiety after spontaneous abortion and also help manage complicated pregnancies.
\end{abstract}

